K.A. van der Hucht, G. Koenigsberger \& P.R.J. Eenens, eds.

\title{
The stellar content of NGC 3603
}

\author{
Laurent Drissen \\ Département de Physique and Observatoire du Mont Mégantic, \\ Université Laval, Québec, Qc G1K 7P4, Canada
}

\begin{abstract}
At the heart of the galactic giant $\mathrm{H}$ II region NGC 3603, low-mass $\left(1-4 \mathrm{M}_{\odot}\right)$ stars slowly contract toward the main sequence, while peacefully cohabiting with the three most luminous, hydrogen-rich Wolf-Rayet stars in the Galaxy, a dozen hot O3-4 stars and their more modest but numerous $\mathrm{O}$ and $\mathrm{B}$ companions. This is the story of a very young and dense starburst cluster ...
\end{abstract}

\section{Introduction: NGC 3603 in context}

NGC 3603 is the most luminous optically visible starburst region in our Galaxy. It consists of a giant $\mathrm{H}$ II region of diameter $50 \mathrm{pc}$, with the young compact cluster of the same name at its core. With $\mathrm{L}(\mathrm{H} \alpha) \simeq 1.5 \times 10^{38} \mathrm{erg} \mathrm{s}^{-1}\left(\log N_{\mathrm{Ly}}=50.05\right)$, NGC 3603 is about twice as luminous as the Carina complex and 100 times more luminous than the Orion nebula. But star formation is not as spectacular in the Milky Way as it is in other galaxies, and NGC 3603 pales in comparison with extra-galactic giant $\mathrm{H}$ II regions such as 30 Doradus or NGC 2363 (which are about ten times brighter).

The ionizing cluster of NGC 3603 is so compact that its core has its own HD number: HD 97950 (=WR 43). Most of its members are confined within a radius of $15^{\prime \prime}$, or half a parsec (Figure 1), and the entire cluster is surrounded by an expanding wind-blown bubble (Clayton 1990 and references therein). The rich, dense stellar population in the core of NGC 3603 is very young (less than 2 Myrs) and likely to be coeval; it thus provides a unique opportunity to study the evolution of the very most massive stars. NGC 3603 is also the closest $(7 \mathrm{kpc})$, truly dense starburst where, because of its proximity and ease of study with HST or ground-based adaptive optics, extreme environmental influences on star formation can be studied effectively and unambiguously. NGC 3603 was, until recently, thought to be unique in the Galaxy. But the discovery and detailed study of two similar compact clusters near the galactic center (Figer, these Proceedings) attest to the contrary.

I summarize in this paper our current knowledge about the stellar content of NGC 3603. I would also like to refer the reader to two poster papers on NGC 3603 included in these Proceedings: Devost \& Leitherer present HST-NICMOS images of the core of the cluster, while Tapia \& Pérez show ground-based infrared images of its immediate surroundings.

\section{Resolving the core}

The ionizing cluster of NGC 3603 is very compact, as Figure 1 testifies. Colormagnitude diagrams of the stars at its periphery have been used to estimate its 


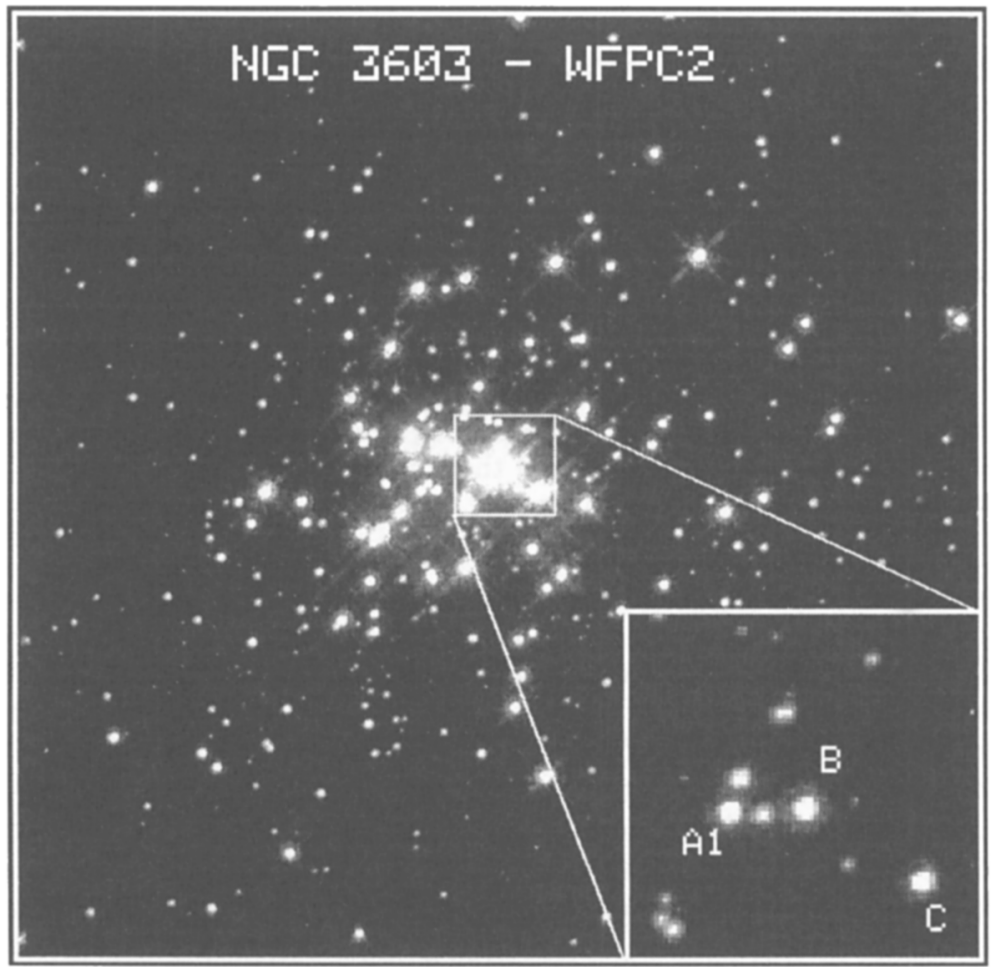

Figure 1. HST-WFPC2 I-band (F814W) image of NGC 3603. The image is $25^{\prime \prime} \times 25^{\prime \prime}(0.8 \mathrm{pc} \times 0.8 \mathrm{pc})$. The insert shows the three WR stars.

distance and age (Melnick et al. 1989), and narrow-band imagery has hinted at the presence of at least two WR stars in the core of the cluster (Moffat et al. 1985), but the true distribution of stars down to the very core has been revealed only recently. Moffat et al. (1994), using the pre-refurbished WF/PC aboard Hubble, and Hofmann et al. (1995), with ground-based speckle interferometry, have spatially resolved the inner region of the cluster. These studies identified three intrinsically very bright WR stars $\left(M_{b}\right.$ ranging from -7.3 to -7.8$)$ within $\sim 1^{\prime \prime}(0.034 \mathrm{pc})$ of the cluster center (stars A1, B and C in Figure 1) and showed that the nature and distribution of the bright stars make NGC 3603 appear as a clone of the central dense cluster R 136 at the heart of 30 Dor in the LMC.

Better quality images aiming at the faint end of the luminosity function will be discussed in section 4 .

\section{Spectroscopic dissection}

The integrated spectrum of HD 97950 is very similar to that of R 136: both spectra are dominated by emission lines of WN5-6 stars obviously diluted by the continuum and absorption lines of early O stars (Walborn 1973; Moffat 1983). 


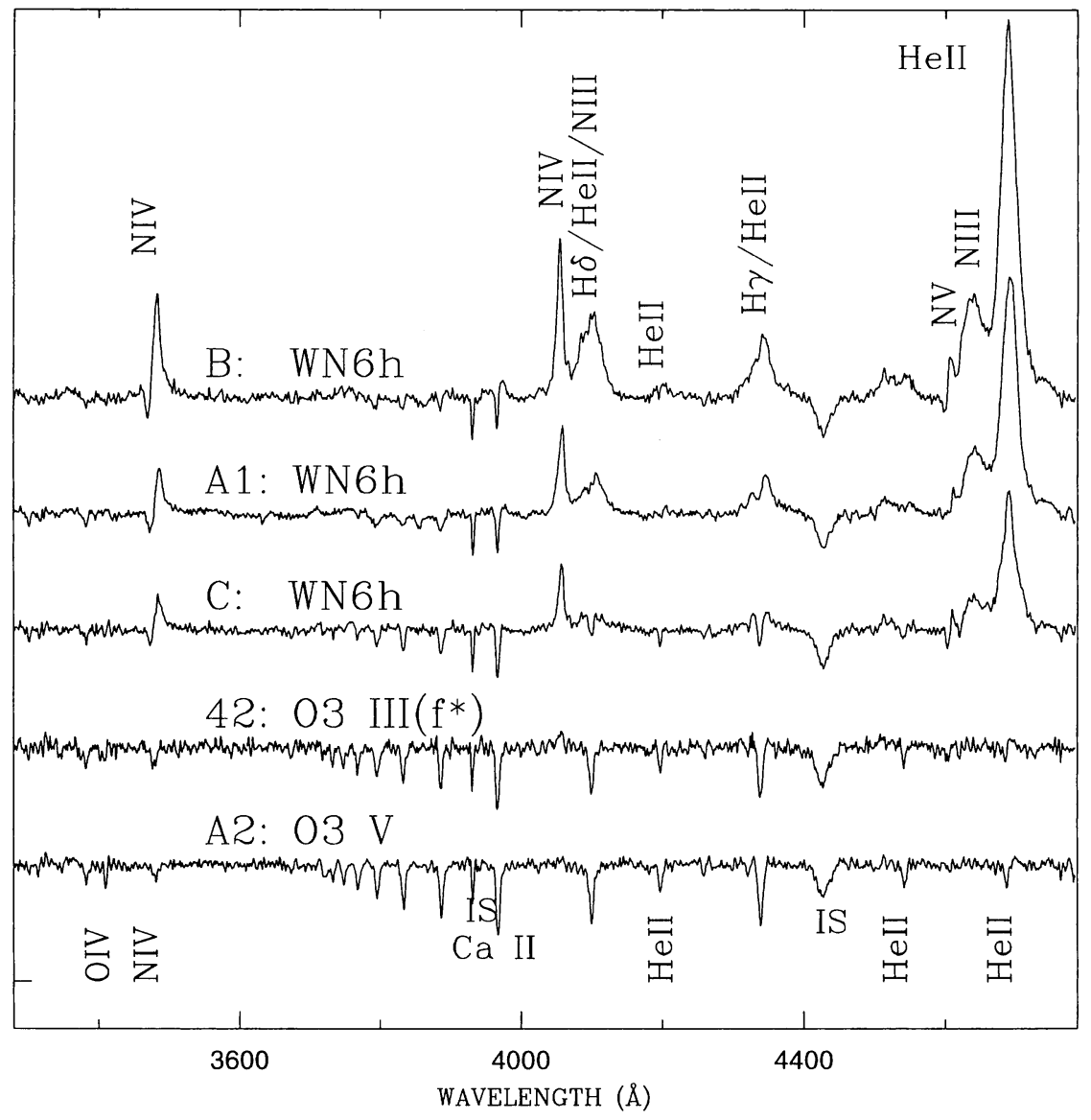

Figure 2. HST-FOS spectrograms of the three WR and two O3 stars in the core of NGC 3603. Note the evolution of the spectral features (He II $\lambda 4686$, $\mathrm{H} \gamma$ and $\mathrm{N}$ IV $\lambda 3485$ for instance) as the luminosity and wind strength increase from $\mathrm{O} 3 \mathrm{~V}$ to O3III to WN6h.

Moffat \& Niemela (1984) have even shown that the WR star which contributes most to the emission spectrum is a binary with a period of 3.8 days.

Because of the high density of the cluster, obtaining uncontaminated spectrograms of individual stars in the core of NGC 3603 is a difficult task. The most recent effort to spectroscopically dissect the cluster was carried out in two phases. Drissen et al. (1995) have obtained spectrograms of 14 bright stars in the very core of NGC 3603 with the HST-FOS. Spectrograms of the five (visually) brightest stars in their sample are shown in Figure 2. It was originally thought that the sequence O3 V $\rightarrow$ O3III $\left(\mathrm{f}^{\star}\right) \rightarrow$ WN6h in this cluster was an evolutionary one, but as Peter Conti first suggested, it appears more likely that the evolution of the spectral features in this particular case simply reflects a difference in luminosity, and hence mass-loss rate. These FOs data also show that the lines in the spectrum of star B are systematically shifted by $\sim 150 \mathrm{~km} \mathrm{~s}^{-1}$ with respect to the same lines in the spectrum of the other WR stars; this sug- 
Table 1. Census of stars with known spectral type in NGC 3603

\begin{tabular}{lccc}
\hline spectral type & number of stars & $N_{\text {Lyc }}$ & $L_{\mathrm{w}}\left(\mathrm{erg} \mathrm{s}^{-1}\right)$ \\
\hline WN6h & 3 & $1.8 \times 10^{50}$ & $1.8 \times 10^{38}$ \\
O3-4 & 15 & $1.3 \times 10^{51}$ & $1.7 \times 10^{38}$ \\
O5 & 7 & $2.8 \times 10^{50}$ & $3.6 \times 10^{37}$ \\
O6 & 2 & $3.4 \times 10^{49}$ & $4.2 \times 10^{36}$ \\
O7 & 1 & $1.0 \times 10^{49}$ & $8.4 \times 10^{35}$ \\
O8 & 1 & $5.4 \times 10^{48}$ & $1.3 \times 10^{35}$ \\
& & & \\
\hline & 29 & $1.8 \times 10^{51}$ & $3.9 \times 10^{38}$ \\
\hline
\end{tabular}

gests that star B is the 3.8 day binary detected by Moffat \& Niemela (1984). Moffat and Walborn and their collaborators recently obtained spectrograms of about a dozen, relatively isolated stars at the periphery of the cluster with the multi-fiber spectrograph ARGUS at CTIO, discovering another half-dozen O3-4 stars. Repeated observations have not yet revealed any obvious binary among this sample. We should note here that the core of the 30 Doradus nebula also contains luminous, H-rich WR stars as well as a large number of very early O3-4 stars (Massey \& Hunter 1998).

The census of the members of NGC 3603 with known spectral type is presented in Table 1, along with the number of ionizing photons they emit, $N_{\mathrm{Lyc}}$, and their wind luminosity, $L_{\mathrm{w}} . N_{\mathrm{Lyc}}$ is taken from Leitherer $(1990) ; L_{\mathrm{w}}$ has been determined using the average values of mass loss rate and terminal velocity for the corresponding spectral type from Howarth \& Prinja (1989).

Two important conclusions can be drawn from the data presented in Table 1. First, the UV flux necessary to ionize NGC 3603 can easily be accounted for by the stars already observed spectroscopically. And since the spectroscopic census is far from complete, we must conclude that a significant fraction of ionizing UV photons produced by the central massive stars is either absorbed by dust within the nebula, or escapes from it. We also note that there is a huge discrepancy (two orders of magnitude!) between the mechanical energy produced by stellar winds and the value determined from the kinematics of the ionized gas (Clayton 1986).

\subsection{The Wolf-Rayet stars}

The only stars in NGC 3603 for which the currently available spectra allow a reliable determination of physical parameters are the three WR stars. Crowther \& Dessart (1998) performed a quantitative analysis of the FOS spectra using John Hillier's detailed non-LTE code. Schmutz \& Drissen (1999) have also analyzed these spectra with a different code (which includes the effects of lineblanketing), but have also used another important piece of information: the 
Table 2. Stellar parameters of Wolf-Rayet stars in NGC 3603. (CD: Crowther \& Dessart 1998; SD: Schmutz \& Drissen 1999)

\begin{tabular}{lrrrr}
\hline HD 97950 & A1 & B & C & ref. \\
& & & & \\
\hline$T_{\text {eff }}(\mathrm{kK})$ & 38 & 46 & 36 & $\mathrm{SD}$ \\
$T_{\text {eff }}(\mathrm{kK})$ & 37 & 35 & 39 & $\mathrm{CD}$ \\
$R_{*}\left(\mathrm{R}_{\odot}\right)$ & 26 & 25 & 23 & $\mathrm{SD}$ \\
$R_{*}\left(\mathrm{R}_{\odot}\right)$ & 30 & 33 & 23 & $\mathrm{CD}$ \\
$\log \left(L / \mathrm{L}_{\odot}\right)$ & 6.1 & 6.4 & 5.9 & $\mathrm{SD}$ \\
$\log \left(L / \mathrm{L}_{\odot}\right)$ & 6.2 & 6.2 & 6.1 & $\mathrm{CD}$ \\
$\log \dot{M}\left(\mathrm{M}_{\odot} \mathrm{yr}^{-1}\right)$ & -4.0 & -3.8 & -4.5 & $\mathrm{SD}$ \\
$\log \dot{M}\left(\mathrm{M}_{\odot} \mathrm{yr}^{-1}\right)$ & -4.2 & -4.0 & -4.6 & $\mathrm{CD}$ \\
& & & & \\
\hline
\end{tabular}

equivalent width of the He I $\lambda 10830$, which they derived from a series of $H S T$ NICMOS images obtained with a continuum filter and a filter centered on the He I line. The parameters they derive are very similar to those obtained by Crowther \& Dessart, with the exception of the temperature, and hence luminosity, of star B (see Table 2). Both studies conclude that (a) the WR triplet in NGC 3603 are among the most luminous WR stars known, and are consequently very massive $\left(M_{\text {init }} \geq 100 \mathrm{M}_{\odot}\right)$; and $(b)$ their atmosphere is rich in hydrogen $(\mathrm{H} / \mathrm{He} \simeq 6)$, which implies, given their high mass-loss rate, that they are very young ( $\leq 2 \mathrm{Myr}$ ).

The accuracy of the parameters would be much improved by UV spectroscopy (to derive a precise value of the wind terminal velocity and the abundances of heavy elements) as well as optical spectra in the $\mathrm{H} \beta$ to $\mathrm{H} \alpha$ range.

\subsection{Evolved supergiant stars}

There are three blue supergiant stars in the immediate vicinity of the cluster: Sher 18 (O6If), Sher 23 (O9.5Iab), and Sher 25 (B1.5Iab), all with magnitudes and reddening estimates consistent with them being associated with NGC 3603 . The case of Sher 25 is particularly interesting since the discovery by Brandner et al. (1997) of a bipolar nebula in many respects (shape, size, expansion velocity and nitrogen enhancement) similar to the famous triple ring around SN 1987A.

Given the very young age of the massive WR and O3 stars as well as the low-mass pre-main sequence stars (see below), it is very unlikely that the blue supergiants are part of the present burst. However, as suggested by Brandner et al. (1987), is is possible that the presence of a first generation of massive stars (including Sher 25) has heated and compressed a part of the dense molecular cloud still visible today and hence initiated the burst that we are witnessing now.

\section{What about low-mass stars?}

The current wide-spread wisdom that starburst clusters and galaxies are deficient in low-mass stars $\left(M \leq 3-5 \mathrm{M}_{\odot}\right)$, is mostly based on indirect evidence derived 


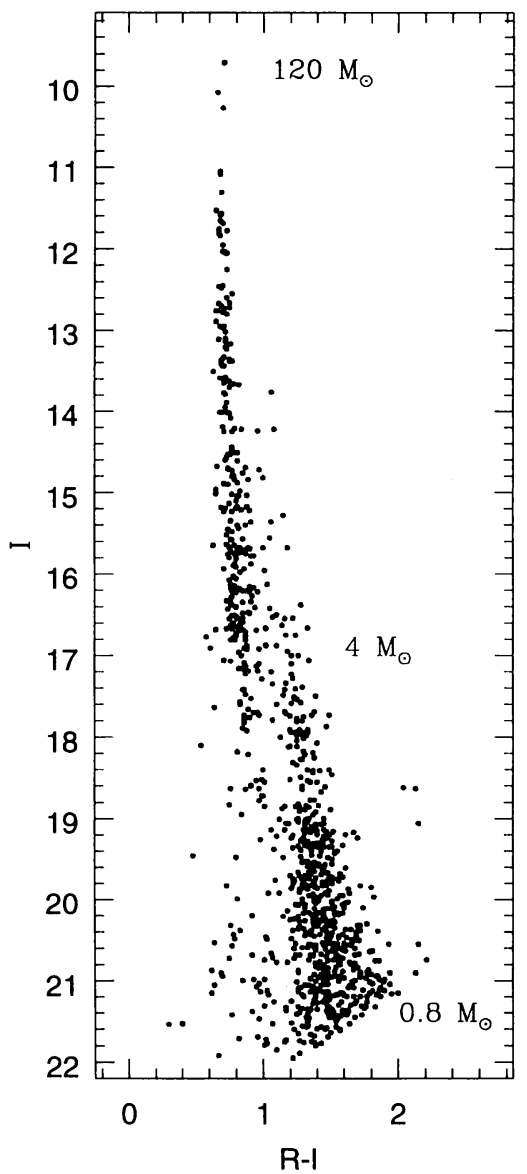

Figure 3. Color-magnitude diagram of the core of NGC 3603, from HSTWFPC2 images.

from the integrated properties of objects like M 82 (Rieke et al. 1993), as well as theoretical considerations (Silk 1995). In collaboration with Moffat and Grebel, I obtained a series of relatively short $(0.5-30 \mathrm{~s})$ VRI images of NGC 3603 with the HST-WFPC2 in order to study its intermediate and low-mass star content. The color-magnitude diagram, which spans 12 magnitudes in $I$ (about a factor of 10 million in luminosity!), is shown in Figure 3; it includes about 1000 stars seen in the high resolution PC CCD.

This diagram shows a narrow main sequence dominated by the three WR stars going down to $I=17$ (corresponding to $M \simeq 4 \mathrm{M}_{\odot}$ ), followed by a pre-main sequence, narrow at first but widened by photometric uncertainties down to the limit determined by the short exposure times. It is important to note that field contamination, as determined from the three wide Field CCDs, is rather low $(\sim 10 \%)$. We still need to fit pre-main sequence evolutionary tracks (with different accretion rates) to the data, but our first attempts indicate that the faintest stars visible on our WFPC2 images are in the range $0.8-1.0 \mathrm{M}_{\odot}$. Our 
study confirms the results of Eisenhauer et al. (1998), based on $J H K$ images obtained with the ADONIS adaptive optics system on the ESO 3.6m telescope, which moreover determine that the pre-main sequence stars are between 0.3 and 1 Myr old. Similar studies on R 136, although less deep and complete than those on NGC 3603 because of its larger distance, also show the presence of a large number of pre-main sequence low-mass stars in the company of many WR and O-type stars (Sirianni et al. 1999).

\section{Conclusions}

- With three luminous Wolf-Rayet stars, more than a dozen O3-O4 and many more $\mathrm{O}$ and $\mathrm{B}$ stars packed in a volume of less than a cubic lightyear, the ionizing cluster of NGC 3603 is one of the densest concentrations of very massive stars known in the Galaxy. NGC 3603, as well as the other clusters recently found and studied close to the Galactic center (Figer, these Proceedings) are the less massive counterparts of the extra-galactic super-star clusters observed in starburst galaxies.

- The three Wolf-Rayet stars at the core of NGC 3603 are not 'classical' WR stars; they are still hydrogen-rich, and mimic the spectrum of WR stars because of their extreme luminosity and mass-loss rate. One of them (very likely star B) is a 3.8-day binary.

- The cluster is much younger than previous estimates based on the presence of WR stars: 1 Myr or less.

- There are more UV ionizing photons produced by the central stars than are required to ionize the surrounding $\mathrm{H}$ II region. Either some of these photons escape the nebula or are trapped by dust.

- A large number of 'low-mass' (1-4 $\left.\mathrm{M}_{\odot}\right)$ pre-main sequence stars are present in the cluster. Therefore, the formation of high-mass stars in a dense starburst does not prevent the formation of low-mass stars.

Acknowledgments. I would like to thank my collaborators on this project: Tony Moffat, Werner Schmutz, Eva Grebel, Mike Shara and Nolan Walborn.

\section{References}

Brandner, W., Grebel, E.K., Chu, Y.-H., Weis, K. 1997, ApJL 475, L45

Clayton, C.A. 1986, MNRAS 219, 895

Clayton, C.A. 1990, MNRAS 246, 712

Crowther, P.A., Dessart, L. 1998, MNRAS 296, 622

Drissen, L., Moffat, A.F.J., Walborn, N.R., Shara, M.M. 1995, AJ 110, 2235

Eisenhauer, F., Quirrenbach, A., Zinnecker, H, Genzel, R. 1998, ApJ 498, 278

Hofmann, K.-H., Seggewiss, W., Weigelt, G. 1995, A\&A 300, 403

Howarth, I.D., Prinja, R.K. 1989, ApJS 69, 527

Leitherer, C. 1990, ApJS 73, 1 
Massey, P., Hunter, D. 1998, ApJ 493, 180

Melnick, J., Tapia, M., Terlevich, R. 1989, A\&A 213, 89

Moffat, A.F.J. 1983, A\&A 124, 273

Moffat, A.F.J., Drissen, L., Shara, M.M. 1994, ApJ 436, 183

Moffat, A.F.J., Niemela, V.S. 1984, ApJ 284, 631

Moffat, A.F.J., Seggewiss, W., Shara, M.M. 1985, ApJ 295, 109

Rieke, G.H., Loken, K, Rieke, M.J., Tamblyn, P. 1993, ApJ 412, 99

Schmutz, W., Drissen, L. 1999, in: N.I. Morrell \& V.S. Niemela (eds.), Hot Stars in Open Clusters of the Galaxy and the Magellanic Clouds, Proc. La Plata Workshop, December 1997, RevMexAA-SC in press

Silk, J. 1995, ApJ 438, L41

Sirianni, M., Nota, A., Leitherer, C., Clampin, M., De Marchi, G. 1998, in: I. Howarth (ed.), Properties of Hot, Luminous Stars, Proc. Boulder-Münich Workshop II, ASP-CS 131, 363,

Walborn, N.R. 1973, ApJL 182, L21

\section{Discussion}

Walborn: Your color-magnitude diagram illustrates an interesting point about coevality in rich young clusters, namely that the massive stars are coeval while the lower masses show a spread. [Followed by Maeder comment about accretion]. Yes, also if the premain-sequence lower masses still have disks, there will be orientation effects in the photometry.

Drissen: The color spread in the lower part of the CM diagram is mostly due to photometric errors, so I am not convinced that we see an age spread.

Maeder: The question of coevality of the low mass pre-MS stars critically depends on the kind of pre-MS tracks you are using, e.g., classical tracks with constant mass or tracks with mass accretion. On the whole, I think your results may be a major test for pre-MS evolution models.

Drissen: I agree. I will send you the data and we will be able to constrain the accretion rate of pre-MS stars.

Leitherer: Did you attempt to relate the X-ray emission to the properties of the stellar population of NGC 3603 ? In particular, are the X-rays due to hot, wind-blown bubbles or do they come from massive X-ray binaries?

Drissen: You-Hua Chu and I are still working on the interpretation of the ROSAT-HRI images, but it seems clear that the X-rays produced by the hot gas within the windblown bubble are not visible due to the large absorption along the line-of-sight. The $\mathrm{X}$-rays we do see have a stellar origin.

Moffat: It is interesting to note the pile-up of O3-type stars both in NGC 3603 and in $\mathrm{R}$ 136. Perhaps our spectra are not of sufficient quality to distinguish very weak $\mathrm{He}$ I 4471 so that one may have to introduce $\mathrm{O} 2$ or $\mathrm{O} 1$ and younger ages. As Nolan says, the O3 type is one-sided, with He I 4471 not seen.

Koenigsberger: (1) Is there any basis for expecting a larger fraction of binaries in such a dense cluster? (2) Isn't it possible that all the most massive stars you detect are really binaries like HD 5980 ?

Drissen: (1) I do not expect an abnormal binary fraction in NGC 3603, although the opinion of an expert in this field would be more welcome. The binary fraction in globular clusters of similar density is $\sim 25-35 \%$. 
(2) Star B is a short-period binary $(P=3.77 \mathrm{~d})$ and it is likely that one of the other WR stars is a longer-period binary. But from the spectra of the other $\sim 25$ stars, we do not have evidence for obvious binaries, which does not mean that there are no binaries.

Tapia: I would like to point out a fact that is relevant when talking about the history of NGC 3603. Within $4 \mathrm{pc}$ North of HD 97950 there are at least five K- and M-type supergiants. This number exceeds by a factor of 8 the number of field stars expected per unit area in that direction. Their association to NGC 3603 needs to be studied.

Drissen: I agree. These red supergiants may also be related to Sher 25, a $15 \mathrm{Myr}$-old blue supergiant, that has already been through a RSG-phase.

van der Hucht: You quote a distance $d($ NGC 3603$)=7 \mathrm{kpc}$ from earlier work by Moffat et al. Crowther \& Dessart (1998) derive $d=10 \mathrm{kpc}$. Is the difference between the error bars or more fundamental?

Drissen: The value of $7 \mathrm{kpc}$ has been determined from $U B V$ photometry of isolated massive stars (Moffat et al.; Melnick et al.). Crowther \& Dessart derive a different value of $E_{B-V}$ (1.2 instead of 1.4) based on the fit to the spectra of WR stars on the cluster. From our VRI images, it appears that the extinction law may be abnormal in the line-of-sight towards NGC 3603. To solve this problem, we need UV to optical spectra of individual $\mathrm{O}$ stars on this cluster.

Terlevich: Do you find evidence for mass segregation in NGC 3603, in the sense that the core radius of the massive stars is different to that of the low-mass stars?

Drissen: The three most massive stars are right at the core of the cluster. We are presently working on the spatial distribution of stars of different masses. The main problem here is to determine the incompleteness levels in the very core, which is a strong function of the radius.

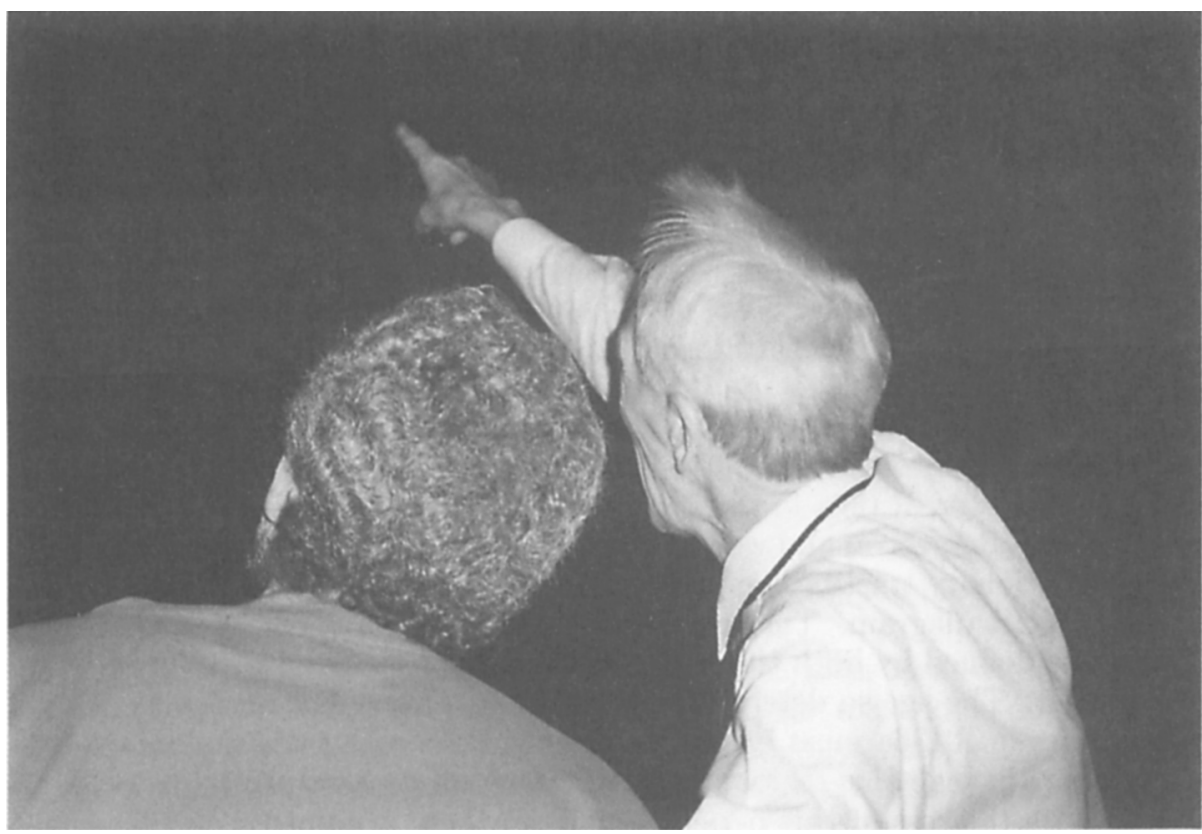

